\title{
Functional Connectivity in Virally Suppressed Patients with HIV-Associated Neurocognitive Disorder: A Resting-State Analysis
}

\author{
(D).R. Chaganti, (D) A. Heinecke, (D)T.M. Gates, (D).J. Moffat, and (D)B.J. Brew
}

\begin{abstract}
BACKGROUND AND PURPOSE: HIV-associated neurocognitive disorder still occurs despite virally suppressive combination antiretroviral therapy. In the pre-combination antiretroviral era and in patients without HIV suppression, HIV-associated neurocognitive disorder was caused by synaptodendritic injury resulting in impairment of neural networks, characterized by decreased attention, psychomotor slowing, and working memory deficits. Whether similar pathogenesis is true for HIV-associated neurocognitive disorder in the context of viral suppression is not clear. Resting-state fMRI has been shown to be efficient in detecting impaired neural networks in various neurologic illnesses. This pilot study aimed to assess resting-state functional connectivity of the brain in patients with active HIV-associated neurocognitive disorder in the context of HIV viral suppression in both blood and CSF.
\end{abstract}

MATERIALS AND METHODS: Eighteen patients with active HIV-associated neurocognitive disorder (recent diagnosis with progressing symptoms) on combination antiretroviral therapy with viral suppression in both blood and CSF and 9 demographically matched control subjects underwent resting-state functional MR imaging. The connectivity in the 6 known neural networks was assessed. To localize significant ROIs within the HIV and control group, we performed a seed-based correlation for each known resting-state network.

RESULTS: There were significant group differences between the control and HIV-associated neurocognitive disorder groups in the salience $(0.26$ versus $0.14, t=2.6978, d f=25, P=.0123$ ) and executive networks $(0.52$ versus $0.32, t=2.2372, d f=25, P=.034)$. The covariate analysis with neuropsychological scores yielded statistically significant correlations in all 6 studied functional networks, with the most conspicuous correlation in salience networks.

CONCLUSIONS: Active HIV-associated neurocognitive disorder in virally suppressed patients is associated with significantly decreased connectivity in the salience and executive networks, thereby making it potentially useful as a biomarker.

ABBREVIATIONS: CART = combination antiretroviral therapy; $\mathrm{HAND}=\mathrm{HIV}$-associated neurocognitive disorder; $\mathrm{NC}=$ neurocognitive; rs-fMRI $=$ resting-state fMRI

$\mathbf{H}^{\mathrm{i}}$

IV-associated neurocognitive disorder (HAND) persists in the era of potent combination antiretroviral therapy (cART), even when confounding conditions are excluded and patients are virally suppressed. ${ }^{1,2}$ HAND is a broad term to encompass neurocognitive disorders including asymptomatic neurocognitive impairment, mild neurocognitive impairment, and HIV-associated dementia with prevalence ranging from $18 \%$ to $52 \%$. The diagnosis of different stages of HAND is based on the recommen-

Received July 11, 2016; accepted after revision March 29, 2017.

From the Departments of Radiology (J.R.C., K.J.M.) and Neurology (B.J.B.), St Vincent's Hospital, Darlinghurst, Sydney, New South Wales, Australia; Brain Innovation B.V. (A.H.), Maastricht, the Netherlands; Department of Neurology, Clinical Research Program (T.M.J.) and Neurosciences Program, Peter Duncan Neurosciences Unit (B.J.B.), St Vincent's Centre for Applied Medical Research, University of New South Wales, Sydney, Australia.

Please address correspondence to Joga Chaganti, MD, St Vincent's Hospital, Victoria Street, Darlinghurst, Sydney 2100, Australia; e-mail: joga.chaganti@svha.org.au

http://dx.doi.org/10.3174/ajnr.A5246 dations from updated research nosology for HIV-associated neurocognitive disorders ${ }^{1}$ and includes neuropsychological testing and neurologic evaluation. The variation in the prevalence depends on whether patients with confounding conditions and unsuppressed HIV viral load are included. Nonetheless, even in the absence of confounds and the presence of viral suppression in both blood and CSF, HAND still occurs and may develop for reasons that are unclear. ${ }^{3,4}$

HAND is characterized by decreased attention, psychomotor slowing, and working memory deficits. ${ }^{5,6}$ While HAND in the pre-cART era was characterized as subcortical, the advent of CART has shifted the impairment to include higher order cognitive abilities such as executive functioning. ${ }^{3,5}$ The higher order cognitive dysfunction was substantiated by a number of taskbased functional MR imaging studies that demonstrated increased use of reserve brain regions during working memory tasks in patients with HIV with mild dementia and in those 
who were neuroasymptomatic. ${ }^{7,8}$ These studies showed that there are abnormal levels (initial increase and later reduced activity) of activation in the dorsolateral prefrontal cortex and parietal cortices. However, these studies did not include patients who were virally suppressed in both blood and CSF.

Resting-state fMRI (rs-fMRI) has been established as a powerful tool to measure the brain networks, which are implicated in cognitive disorders. Currently, there are only 5 studies that have examined resting-state networks in HAND. ${ }^{9-13}$ These studies have noted abnormalities, especially early in the course of HIV infection. ${ }^{9}$ Some studies have shown conflicting results (Ipser et $\mathrm{al}^{11}$ and Guha et $\mathrm{al}^{12}$ ). However, no studies have been specifically designed to address the pathogenesis of HAND in the cART era. Thus, additional criteria are required. Patients with HAND must be virally suppressed in both blood and CSF and should demonstrate symptoms of HAND that are recent and progressive and are not related to the legacy effect from the past (cognitive abnormalities that reflect "burn-out" disease).

Consequently, the aim of this study was to analyze the effect of HIV infection on various resting networks in virally controlled patients with active HAND as a means of understanding the pathogenesis in the cART era.

\section{MATERIALS AND METHODS}

Eighteen patients with HIV with HAND were enrolled. All had been on a stable cART regimen for at least the previous 12 months, and all had HIV RNA levels of $<20$ copies per $\mathrm{mL}$ in both blood and CSF. All had recent onset of symptoms within the past 6 months. All were assessed by a neurologist, and all had blood and CSF analyses to exclude other causes of cognitive impairment. All had $\beta-2$ microglobulin and neopterin measured in both blood and CSF. (Both $\beta$-2 microglobulin and neopterin are markers of immune activation and inflammation and are known to be elevated in many neuroinflammatory conditions and are shown to be mildly elevated in patients who have suppressed plasma and CSF HIV RNA to below the detection limits of clinical assays). All were diagnosed with HAND by the American Academy of Neurology criteria, ${ }^{14}$ with a full neuropsychological assessment. ${ }^{15,16}$ Of these 18 patients with HAND, 12 were identified with mild neurocognitive impairment (12/18); 4 patients, with asymptomatic neurocognitive impairment; and 2, with HIV-associated dementia (2/18). Individuals with a prior history of drug abuse, significant head injury, psychiatric illness, and Hepatitis C co-infection were excluded. Nine HIV seronegative matched controls were included in the study. None of the patients had any white matter abnormalities or microhemorrhages on anatomic MR imaging protocols. Local ethics approval was obtained from the St Vincent's Hospital Human Research Ethics Committee, and all the participants provided written informed consent before enrollment (Table 1).

\section{Neurocognitive Evaluation}

Neurocognitive testing was performed with a brief research test battery described previously, which has been validated for use in the HIV-positive population. ${ }^{14,15}$ Briefly, the test battery consisted of a brief computerized battery (CogState) supplemented with a small selection of standardized pencil-and-paper tests to
Table 1: Demographic, HIV, and neurocognitive characteristics of the study sample

\begin{tabular}{lccc}
\hline & HIV+ $(\boldsymbol{n}=\mathbf{1 8})$ & $\begin{array}{c}\text { Control } \\
(\boldsymbol{n}=9)\end{array}$ & $\boldsymbol{P}$ \\
\hline Age (mean) (SD) (yr) & $53.7(7.5)$ & $55(4.0)$ & .6897 \\
Male sex (\%) & 100 & 100 & \\
Ethnicity (\% white) & 100 & 100 & \\
Education (mean) (SD) (yr) & $13.0(3.0)$ & $15(2.0)$ & .0043 \\
Duration stable on cART & $2(2)$ & $\mathrm{NA}$ & \\
(median) (IQR) (yr) & & & \\
CD4 cell count (median) & $657(550)$ & & \\
$\quad$ (IQR) (cells/mm (m) $^{3}$ & & & \\
Nadir CD4 (median) (IQR) & $194.5(263.5)$ & \\
Composite neurocognitive & $-0.69(0.75)$ & \\
z score (median) (IQR) & & \\
\hline Note:-NA indicates not applicable; IQR, interquartile range; +, positive.
\end{tabular}

Table 2: Tests included in the brief neurocognitive research battery

\begin{tabular}{cl}
\hline \multicolumn{1}{c}{ Cognitive Domain } & \multicolumn{1}{c}{ Tests } \\
\hline $\begin{array}{c}\text { Speed of information } \\
\text { processing }\end{array}$ & $\begin{array}{l}\text { CogState Detection Task } \\
\text { CogState Identification Task } \\
\text { Trail-Making Test: Parts A and B } \\
\text { WAIS-III Digit Symbol Coding }\end{array}$ \\
Attention/working memory & $\begin{array}{l}\text { CogState One Back Task } \\
\text { CogState Two Back Task } \\
\text { Motor functioning }\end{array}$ \\
Gerbal learning & $\begin{array}{l}\text { Non-Dominant Hand } \\
\text { CogState International Shopping } \\
\text { List Task: Learning }\end{array}$ \\
Verbal memory & $\begin{array}{c}\text { CogState International Shopping } \\
\text { List Task: Delayed Recall }\end{array}$ \\
\hline
\end{tabular}

Note:-WAIS-III indicates Wechsler Adult Intelligence Scale-III.

cover 5 cognitive domains: 1) speed of information processing, 2) attention/working memory, 3 ) motor coordination, 4) verbal learning, and 5) verbal memory (Table 2). CogState is sensitive to $\mathrm{HAND}^{17}$ and uses playing cards as stimuli to minimize the influences of culture and education on testing. Other tests were selected to provide greater coverage of other cognitive domains affected by HAND (psychomotor speed and motor coordination). Additionally, premorbid intellectual functioning was estimated with the National Adult Reading Test or a demographic-based regression equation when the National Adult Reading Test could not be validly administered. Psychological measures included the Depression, Anxiety, and Stress Scales and the Mini-International Psychiatric Interview (Version 5.0.). Functional decline for HAND status classification was measured via a standard Independence of Activities of Daily Living questionnaire. ${ }^{18}$

\section{Image Acquisition}

All imaging was performed with a 3T MR imaging scanner (Ingenia; Philips Healthcare, Best, the Netherlands) with a 24-channel head coil. High-resolution 3D T1-weighted volume and rs-fMRI scans were obtained. Volumetric scanning was performed with T1 fast-field echo (TR, $7600 \mathrm{~ms}$ [shortest]; TE, $3.5 \mathrm{~ms}$; bandwidth, 191.5 Hz/per pixel; flip angle, $7^{\circ}$; acquisition matrix, 256/256; section thickness, $1-\mathrm{mm}$ isometric voxel [3D encoding] with an acceleration of $2 \times$ sensitivity encoding).

We used a resting-state scan sequence in which participants were instructed to focus on a white cross presented on a screen 
during the scan. They were also asked not to think of anything in particular. The parameters were the following: total duration, 7.39 minutes; TR/TE, 3000/35 ms; flip angle, 90 150 dynamics with the shortest dynamic scan time; acquisition matrix, 230/230/ 94; section thickness, $4 \mathrm{~mm}$ acquired parallel to the anterior/posterior commissure plane.

Preprocessing. The data processing was performed on BrainVoyager QX, Version 2.8.4 (Brain Innovation, Maastricht, the Netherlands). Anatomic images were corrected for inhomogeneity with an automatic intensity-based approach (which included a brain extraction). The functional data were corrected for the differences in section scan-time acquisition, motion in 3 planes (with the first volume as reference), and linear and nonlinear temporal drifts with a high-pass filter based on a general linear model with a Fourier basis set (1 linear, 2 sine, and 2 cosine functions). Native functional and anatomic data were coregistered in 2 steps with first, the header-based positioning information and, second, an intensity-based alignment algorithm.

Image Analysis. Subsequently both the anatomic and functional data were normalized with affine transformation into the anterior/posterior commissural planes and finally to Talairach space with an inbuilt function of BrainVoyager, which uses a piecewise linear approach. ${ }^{19,20}$

On the basis of the standardized (Talairach) 3D anatomic dataset, white matter and ventricle voxels were defined for each subject. From both anatomic areas, the average time courses were extracted and used in combination with motion confounds (6 parameters) to regress out specific sources of noise within the functional data. ${ }^{21}$ ROI time-series were computed by selecting a seed location based on predefined coordinates in the Talairach Atlas (based on the Brodmann file available in the BrainVoyager standard installation) and taking the average of all voxels from the following ROIs: Brodmann areas 2 (somatosensory cortex-network), 8 (intraparietal sulcus-dorsal attentional networks), 9 (dorsolateral prefrontal cortex-executive network), 13 (anterior insular cortex-salience network), 17 (primary visual cortex-visual network), and 31 (posterior cingulate-default mode network) based on a predefined/standard ROI file in Talairach space.

Postprocessing and Statistical Analysis. To localize significant ROIs within the HIV and control groups, we performed a seedbased correlation for each of the 6 mentioned resting-state networks. On the basis of subject-specific correlation maps, an independent $t$ test was performed to show up the differences between the HIV and control groups. The analysis of the comparison of interregional connectivity between the HIV and control group included the following steps: Consistent regions between both the groups were defined for each resting-state network on the basis of the anatomic conjunction (overlap) of functional maps. From these overlapping ROIs in each resting-state network, the signal time courses were extracted for each subject. Pair-wise correlations were calculated from within each network, and the average correlations were computed at the subject level. On the basis of the subject-specific inter-region correlations, an independent $t$ test was used to assess the differences between the HIV and con- trol groups. All statistical analysis was performed with SPSS (Version: 1.2; IBM, Armonk, New York).

\section{Inclusion of External Covariates}

A single composite neurocognitive $z$ score was generated through 2 steps. First, CogState normative data raw scores were converted to age-corrected $z$ scores $(\mathrm{M}=0, \mathrm{SD}=1)$ with CogState normative data, and raw scores on other tests were converted to ageadjusted scaled scores with published normative data ${ }^{14}$ before being transformed into $z$ scores. ( $Z$ scores are standardized scores, ie, transformed to fit in a normal curve after being adjusted for variables such as age and sex. In healthy individuals, the distribution of standardized cognitive scores, after adjustments, is centered at zero [mean and median] with an SD of 1. Cognitively impaired individuals may have a cognitive score lower than that of healthy individuals, so the average $z$ score for cognitively impaired individuals is much lower). Second, the average of each subset $z$ score was taken to derive a composite neurocognitive score for each patient (Table 1).

The neurocognitive (NC) scores of all the 18 patients were plotted, and a median split was performed. On the basis of the median split of the NC score, a 1-way analysis of variance was performed with the seed-based resting-state data as a dependent variable.

Within the resulting $F$-maps, a contrast checking of the difference between group 2 ( $\mathrm{NC}$ score $>$ median) and group 1 (NC score $<$ median) was performed. All resulting $t$ maps were corrected for multiple comparison problems with the cluster-correction approach (Forman et al, 1995 ${ }^{22}$ ) based on an uncorrected starting threshold of $P=.05$ (a classic Forman cluster-correction approach to 3D statistical maps is implemented in BrainVoyager; http://support.brainvoyager.com/documents/Functional_ Statistics/ClusterThreshPluginHelp/). ${ }^{21}$

\section{RESULTS}

rs-fMRI data were obtained from 27 subjects (18 seropositive and 9 seronegative controls). All the participants were included in the final analysis. The controls were of similar age, sex, and ethnicity (Table 1).

\section{Comparisons of Connectivity in Different Seed Region Networks}

There were significant group differences between the average intercluster correlation in the control and patient groups in the executive network $(0.26$ versus $0.14, t=2.6978, d f=25, P=$ .0123 ) and salience network ( 0.52 versus $0.32, t=2.2372, d f=25$, $P=.034$ ), indicating that there is significantly reduced synchronicity within these 2 networks (salience and attentional networks) (Figs 1 and 2; Table 3).

The difference between controls and patients was trending toward being significant in the dorsal attention network ( 0.37 versus $0.24, t=1.83, d f=25, P=.07)$.

The group differences in the somatosensory network $(0.29$ versus $0.36, t=0.179)$, ventral attentional network ( 0.37 versus $0.24, t=0.06)$, and default mode network ( 0.21 versus $0.21, t=$ $0.91)$ did not reach statistical significance. 

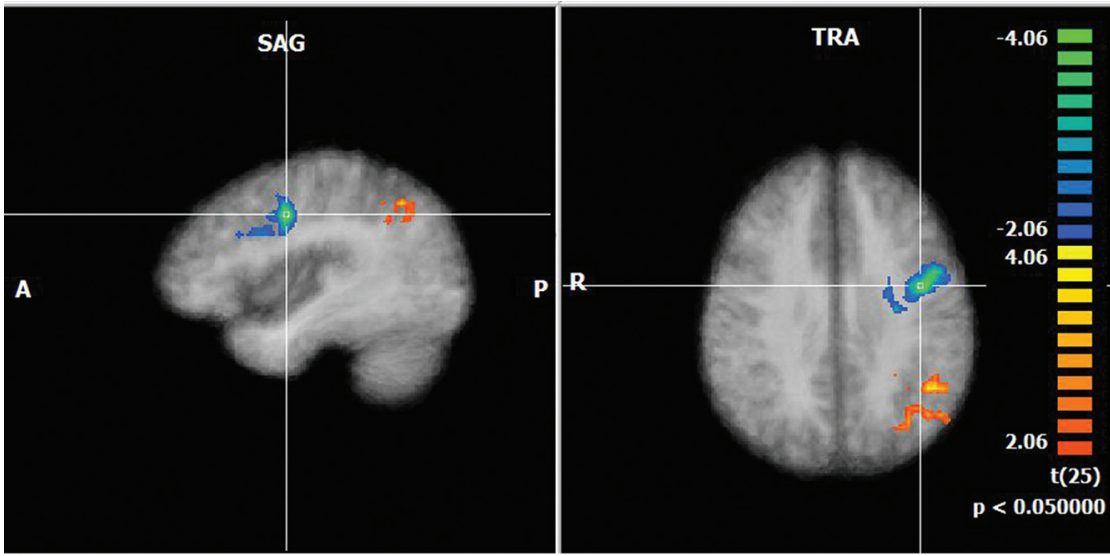

A
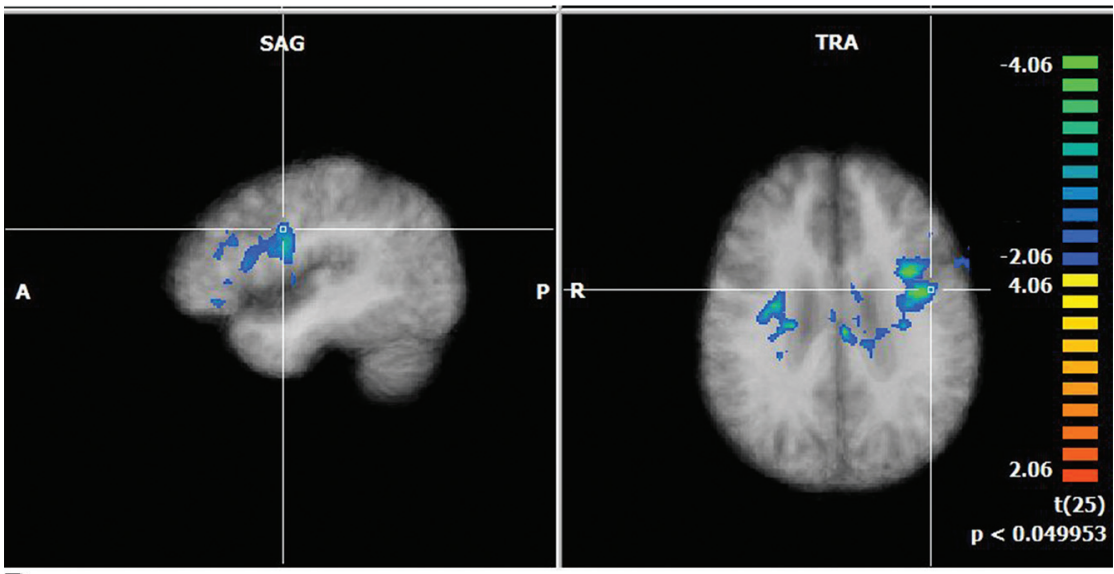

B

FIG 1. Multiplanar and axial depictions of differences between patients with HIV and control subjects in Brodmann area 9 (executive networks) and Brodmann area 13 (salience networks). Positive values represent higher correlation, and negative values depict lower correlation in patients with HIV relative to control subjects. (All the resultant maps are cluster-corrected). A, Brodmann area 9: patients-controls. B, Brodmann area 13: patients-controls.

\section{Correlations between Network Connectivity Analysis and Clinical Measures}

We assessed whether observed changes in brain networks were associated with neuroinflammatory markers (namely CSF and plasma neopterin and $\beta-2$ microglobulin) and NC scores. None of the networks demonstrated any significant positive or negative correlations with plasma/CSF biomarkers. However, neurocognitive scores showed significant correlations with all 6 mentioned networks. Within these networks, the salience networks demonstrated the most significant correlations with reduced neurocognitive scores (Figs 3 and 4).

\section{DISCUSSION}

Our study showed that there is significant attenuation in the salience and executive networks in patients with HAND with active disease despite viral suppression in both plasma and CSF. The network changes correlated with the degree of neuropsychological impairment but not with markers of neuroinflammation in the blood or CSF.

The salience network is considered important for monitoring the salience of external inputs and internal brain events, and the central executive network is engaged in higher order cognitive and attentional control. The salience networks comprise cortical areas of the anterior insula and anterior cingulate and subcortical areas, including the amygdala, substantia nigra, and thalamus. The central executive networks connect the dorsolateral prefrontal cortex to the striatum and posterior inferior parietal areas and have subcortical networks distinct from those in the salience networks. ${ }^{23}$

Only 5 studies examined the effect of HIV and secondarily HAND on rs-fMRI. Foryt et $\mathrm{al}^{9}$ in their study on HIV-positive subjects in their first year of infection showed diminished connectivity within the lateral occipital network (visual attention or visuospatial attention), but there were no patients with HAND. Ipser et $\mathrm{al}^{11}$ had a cohort of 15 HIV-positive patients, 11 of whom were on cART. There were 4 patients with HAND. Reduced frontostriatal connectivity was noted, especially in relation to the executive network. Thomas et $\mathrm{al}^{10}$ had a heterogeneous cohort of 52 HIV-positive patients (23 were on cART but the number who were virally suppressed was not clear and only 12 had HAND). They were evaluated for the effect of aging in HIV compared with seronegative controls. They found attenuation in the default mode and salience networks, similar to that which occurs with age, and inferred that there is premature aging in HIV. This study did not find any correlation with neurocognitive scores or plasma markers.

Guha et $\mathrm{al}^{12}$ evaluated resting networks in HIV-infected individuals older than 60 years of age (HIV-positive, $n=52$, and controls, $n=29$ ). Most were on cART ( $94 \%$ ) but not all were virally suppressed (35 of 50 patients) and 27 had HAND, though none appeared to have active disease. Those with detectable plasma HIV-RNA had reduced connectivity in the salience networks. No significant relationship between the neuropsychiatric performance and rs-fMRI was observed.

Ann et $\mathrm{al}^{13}$ tested the differences in connectivity in seropositive patients with and without HAND, using seed-based connectivity in the default mode network with seed in the precuneus, and demonstrated that there was reduced connectivity between the precuneus and prefrontal regions in the HAND group versus the non-HAND group (10 patients in HAND and 13 in non-HAND). The group separation was not based on CSF viral suppression. The whole-brain networks were not evaluated in this study. It is, therefore, a targeted deterministic seed-based study assessing 1 domain only (default mode network). It is also unclear whether there were any white matter lesions associated with age in this cohort and how much the effect of aging and vascular disease influenced the results.

As previously mentioned, none of the above-mentioned studies included patients with HAND with viral suppression in both 

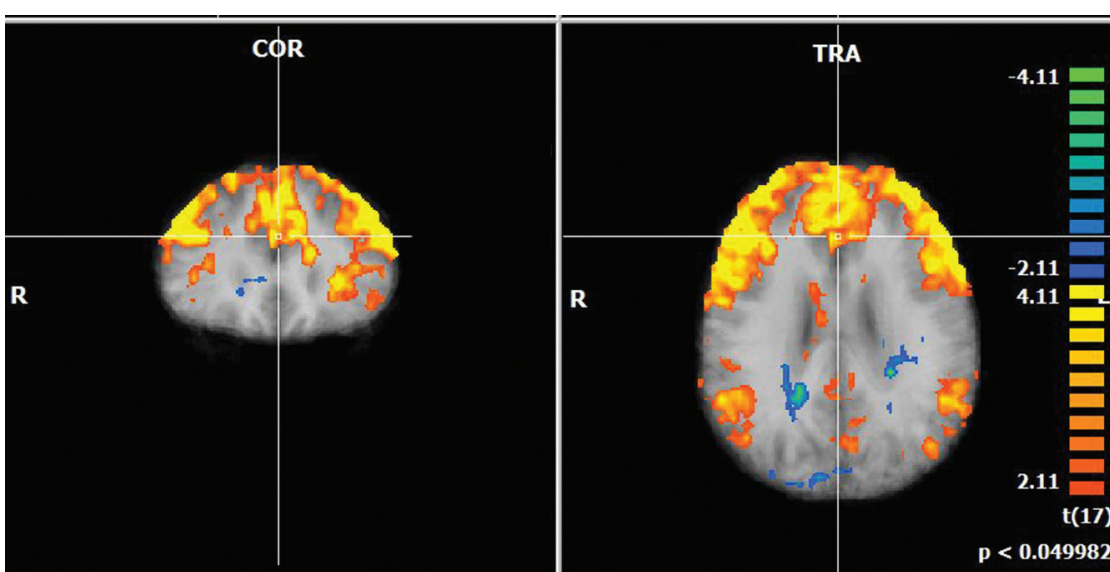

A
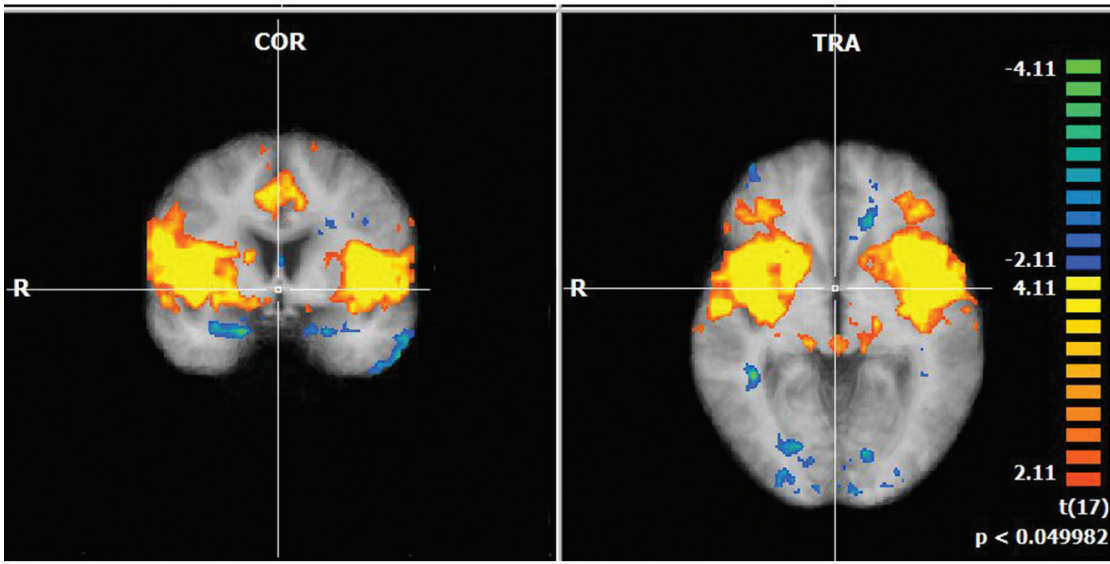

$\bar{B}$

FIG 2. A, Multiplanar depictions of group effect in subjects with HIV in the executive networks with the seed region in Brodmann area 9. Note large consistently activated voxels in the entire group, with activation in the dorsolateral frontal cortex and posterior inferoparietal regions and in the striatum. $B$, Multiplanar depictions of group effect in subjects with HIV in the executive networks with seed regions in Brodmann area 13. Large consistently activated voxels in the group with activation in the anterior insula, anterior cingulate, and thalamus.

Table 3: Mean Talairach coordinates of the center-of-gravity coordinates in X, Y, and Z axes and SD and number of voxels (1-mm resolution of each cluster) in Brodmann area 9 (control executive networks) and 13 (salience networks) seed region networks ${ }^{a}$

\begin{tabular}{lrrrrrrr}
\hline & Mean X & Mean Y & Mean Z & SD X & SD Y & SD Z & Voxel No. \\
\hline BA 9 & & & & & & & \\
Cluster1_conjunction & 5.98 & 25.50 & 34.01 & 35.43 & 23.28 & 11.16 & 78,154 \\
Cluster2_conjunction & 37.79 & -39.71 & 46.68 & 3.68 & 6.20 & 3.33 & 1149 \\
Cluster3_conjunction & 2.67 & 7.61 & 22.38 & 7.57 & 9.84 & 2.31 & 824 \\
Cluster4_conjunction & -2.32 & -65.50 & 37.52 & 4.02 & 3.44 & 5.86 & 1437 \\
Cluster5_conjunction & -32.92 & -61.66 & 49.64 & 6.30 & 5.04 & 3.92 & 1540 \\
BA 13 & & & & & & & \\
Cluster1_conjunction & -30.81 & 10.97 & 12.36 & 14.87 & 15.63 & 13.75 & 16,021 \\
Cluster2_conjunction & 42.25 & 13.22 & 8.38 & 8.56 & 11.14 & 8.12 & 13,973 \\
Cluster3_conjunction & -43.21 & -17.40 & 8.17 & 10.22 & 12.70 & 8.91 & 4252 \\
Cluster4_conjunction & 52.63 & -20.30 & 12.93 & 6.53 & 17.32 & 11.82 & 6609 \\
\hline
\end{tabular}

Note:-BA indicates Brodmann area.

${ }^{a}$ The clusters are defined on the basis of conjointly activated voxels in patients and control subjects. the salience network with an elevated plasma viral load. This may be relevant to our findings: it suggests that active HAND, at least in our study, is associated with viral replication in the brain at a level not reflected in the blood or the CSF.

Our study also identified significant correlations between neuropsychological scores and significant attenuation in all 6 networks, but especially in the salience, default mode, and executive control networks. These could be due to global injury associated with HIV. ${ }^{24,25}$

Our findings may have specificity for HAND. The frontostriatal networks have been found to be attenuated in Parkinson disease, and attenuation of these executive networks along with default mode networks is said to occur when there is coexistent cognitive impairment associated with Parkinson disease. ${ }^{26}$ However, salience network involvement is not known to occur in Parkinson disease. In healthy aging and Alzheimer disease, there is some uniformity in the involvement of default mode networks. In healthy aging, it was found that there is a decline in the connectivity in the default mode networks. It was also found that there is some evidence of increased connectivity with age, possibly as a compensatory mechanism due to changing neural strategies in most of the other networks. Similarly, in Alzheimer disease, there is a decline in connectivity in most of the networks, but this decline is exaggerated with the default mode network being affected most and much more pronounced than in individuals with healthy aging. ${ }^{27}$ Studies in vascular dementia with rs-FMRI showed involvement of multiple domains, with most pronounced changes in the prefrontal posterior cingulate loop, which is not a typical feature in Alzheimer dementia or HAND. ${ }^{28,29}$ Therefore, the demonstration of attenuation of both the executive and salience networks could be useful as a specific biomarker for active HAND.

blood and CSF, and the activity of HAND was not mentioned. Nonetheless, in those studies with patients with HAND, 2 of 3 found a relationship to attenuation of the salience network. ${ }^{10,12}$ Ipser et $\mathrm{al}^{11}$ noted a relationship only with the central executive control network, but the study included patients with substance misuse and only 4 patients had HAND. Of potential additional importance is the finding by Guha et $\mathrm{al}^{12}$ of a reduction in
There are several limitations in interpreting the results of our study. The study group only included white males. Sample sizes used in this study were small; this small size limited the power to detect between-group differences in the salience and attentional networks. Our cohort had a smaller number of controls who did not undergo formal neuropsychological testing though they were entirely asymptomatic. However, the test we used was not a factorial ANOVA and 

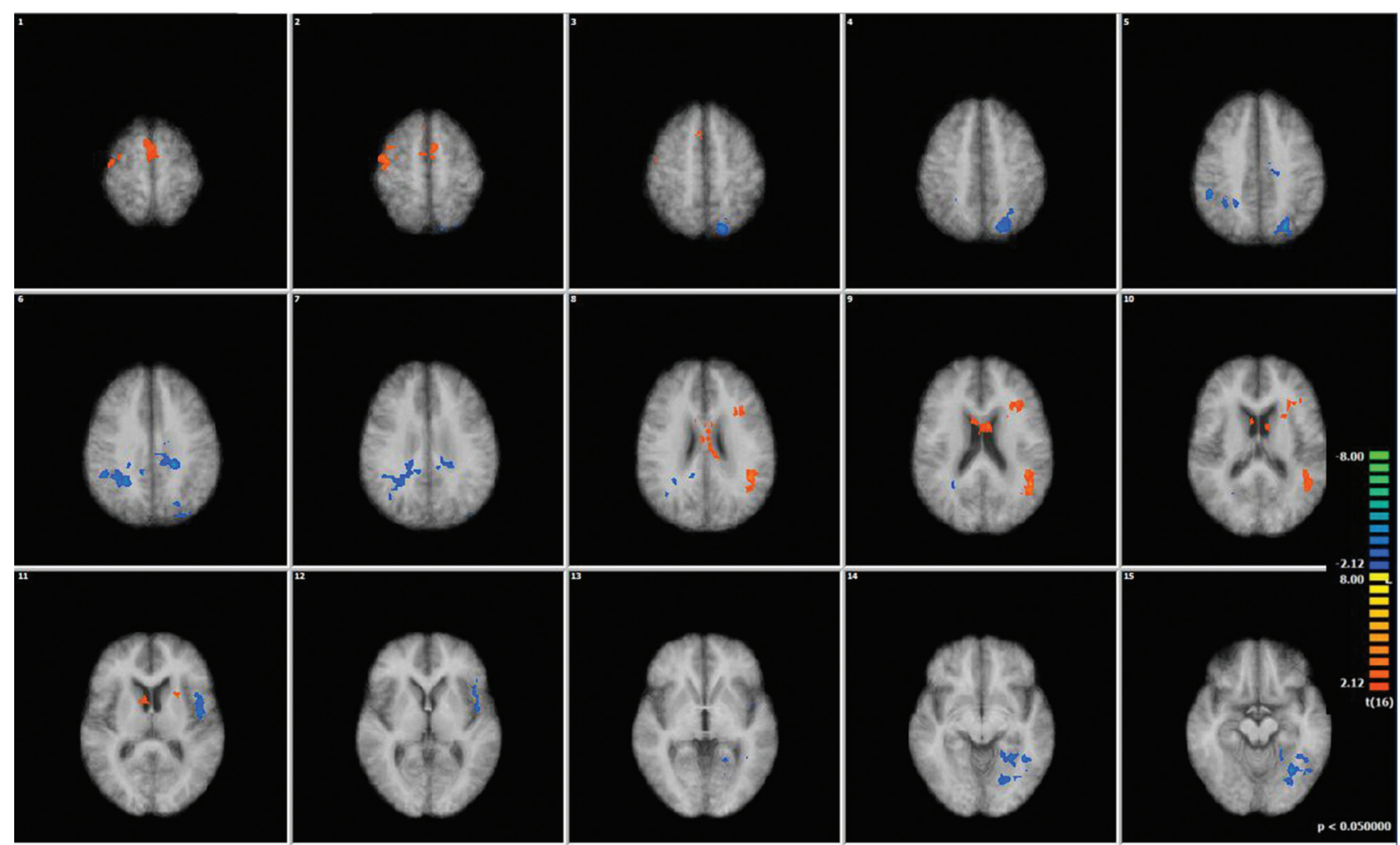

FIG 3. Salience networks. Difference in correlation of the salience network with neurocognitive score (NCS): (Correlation Value in NCS $>$ Median) - (Correlation Value in NCS < median). Orange denotes areas showing larger correlation values in patients with larger neurocognitive values, while blue shows the opposite. Each of the maps is corrected with the cluster-correction approach.

no. of voxels

120000

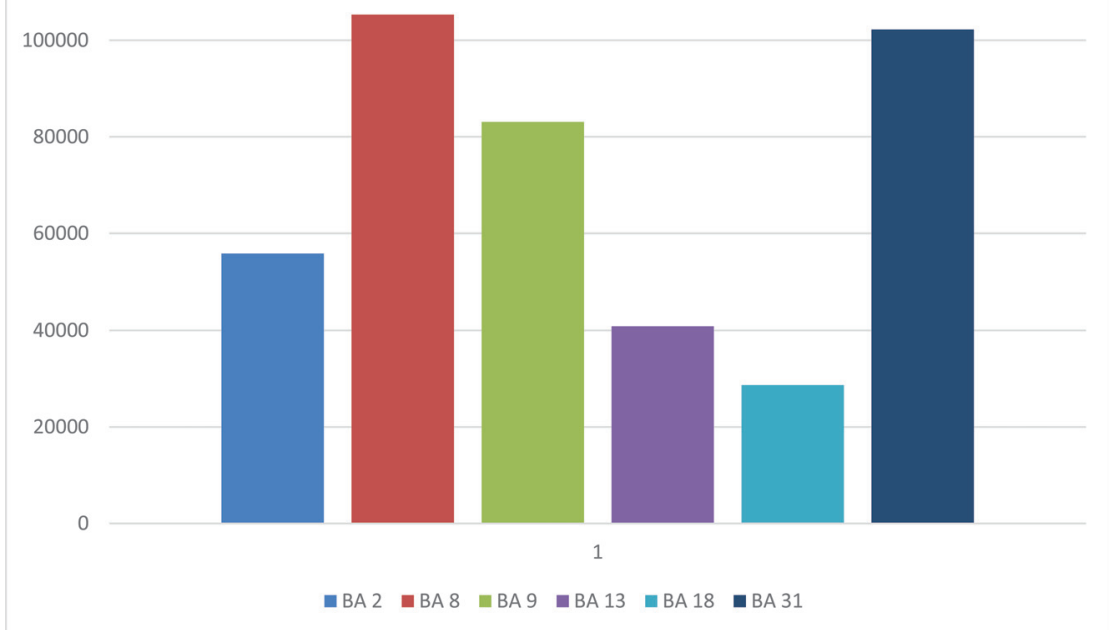

FIG 4. Covariate analysis with neurocognitive scores demonstrating surviving voxels in each of the 6 networks.

hence probably would have a limited effect on the results (we used a $t$ test with heterogeneous variances). In addition, the inherent ambiguities and pitfalls of rs-fMRI could have influenced the outcome of the results through residual effects of noise and inherent blood oxygenation-associated confounds. However, we think these noise effects are not significant because of preprocessing. Last, we cannot exclude the importance of neurotoxicity from cART as opposed to
HIV, given that all the patients in our group were on long-term ( $>4$ years) cART, though the effect of neurotoxicityassociated cognitive impairment appears to be inconclusive. The persistence of the minor cognitive impairment after widespread implementation of cART raised questions about the possible role of the antiretrovirals, particularly nucleoside reverse transcriptase inhibitors. ${ }^{30,31}$ However, several studies have demonstrated the neuroprotective effectiveness of the cART regimen on neurocognitive function. ${ }^{31,32}$ Using MR spectroscopy, investigators have shown that there is marked improvement in both clinical measures and metabolite concentrations. ${ }^{8}$ One recent study used the metrics derived from arterial spin-labeling and rs-fMRI to measure the blood flow and connectivity in HIV-negative individuals before and after using 2 common antiretroviral medications (efavirenz and ritonavir) and observed that there was no effect on either blood flow or connectivity in a small cohort. ${ }^{30,31}$

\section{CONCLUSIONS}

We found significantly decreased connectivity in the executive and salience networks in active HAND, suggesting that it could be used as a biomarker. Furthermore, the association with the sa- 
lience network and its relationship with HIV replication in the blood $^{12}$ raises a further testable and plausible hypothesis: Active HAND in the context of viral suppression in blood and CSF is driven by very low-level brain replication. Future studies will need to validate these findings with larger patient numbers and correlate the findings with CSF single-copy assays of HIV.

Disclosures: Thomas M. Gates_UNRELATED: Travel/Accommodations/Meeting Expenses Unrelated to Activities Listed: ViiV Healthcare Australia, Comments: paid for conference registration: 2014 Association for Psychological Science's College of Clinical Neuropsychologists conference.

\section{REFERENCES}

1. Heaton RK, Clifford DB, Franklin DR Jr, et al; CHARTER Group. $\mathrm{HIV}$-associated neurocognitive disorders persist in the era of potent antiretroviral therapy: CHARTER Study. Neurology 2010;75: 2087-96 CrossRef Medline

2. Heaton RK, Franklin DR, Ellis RJ, et al; CHARTER Group; HNRC Group. HIV-associated neurocognitive disorders before and during the era of combination antiretroviral therapy: differences in rates, nature, and predictors. J Neurovirol 2011;17:3-16 CrossRef Medline

3. Mothobi NZ, Brew BJ. Neurocognitive dysfunction in the highly active antiretroviral therapy era. Curr Opin Infect Dis 2012;25:4-9 CrossRef Medline

4. Cysique LA, Brew BJ. Prevalence of non-confounded HIV-associated neurocognitive impairment in the context of plasma HIV RNA suppression. J Neurovirol 2011;17:176-83 CrossRef Medline

5. Brew BJ. Evidence for a change in AIDS dementia complex in the era of highly active antiretroviral therapy and the possibility of new forms of AIDS dementia complex. AIDS 2004;18(suppl 1):S75-78 Medline

6. Woods SP, Moore DJ, Weber E, et al. Cognitive neuropsychology of HIV-associated neurocognitive disorders. Neuropsychol Rev 2009; 19:152-168 CrossRef Medline

7. Chang L, Tomasi D, Yakupov R, et al. Adaptation of the attention network in human immunodeficiency virus brain injury. Ann $\mathrm{Neu}$ rol 2004;56:259-72 CrossRef Medline

8. Chang L, Yakupov R, Nakama H, et al. Antiretroviral treatment is associated with increased attentional load-dependent brain activation in HIV patients. J Neuroimmune Pharmacol 2008;3:95-104 CrossRef Medline

9. Foryt $\mathrm{P}$, Wang $\mathrm{X}$, Ochs R, et al. Diminished resting-state functional connectivity in lateral occipital cortex in early HIV infection. In: Proceedings of the Annual Meeting of the International Society for Magnetic Resonance in Medicine, Montreal, Quebec, Canada. May 6-13, 2011;19:4163

10. Thomas JB, Brier MR, Snyder AZ, et al. Pathways to neurodegeneration: effects of HIV and aging on resting-state functional connectivity. Neurology 2013;80:1186-93 CrossRef Medline

11. Ipser JC, Brown GG, Bischoff-Grethe A, et al; Translational Methamphetamine AIDS Research Center (TMARC) Group. HIV infection is associated with attenuated frontostriatal intrinsic connectivity: a preliminary study. J Int Neuropsychol Soc 2015;21:203-13 CrossRef Medline

12. Guha A, Wang L, Tanenbaum A, et al. Intrinsic network connectivity abnormalities in HIV-infected individuals over age 60. J Neurovirol 2016;22:80-87 CrossRef Medline

13. Ann HW, Jun S, Shin NY, et al. Characteristics of resting-state functional connectivity in HIV-associated neurocognitive disorder. PLoS One 2016;11:e0153493 CrossRef Medline

14. Antinori A, Arendt G, Becker, et al. Updated research nosology for HIV-associated neurocognitive disorders. Neurology 2007;69:1789-99 CrossRef Medline
15. Heaton RK. Revised Comprehensive Norms for an Expanded HalsteadReitan Battery: Demographically Adjusted Neuropsychological Norms for African American and Caucasian Adults, Professional Manual. Lutz, Florida: Psychological Assessment Resources; 2004

16. Gates TM. Cysique, LA, Siefried, KJ, et al. Maraviroc-intensified combined antiretroviral therapy improves cognition in virally suppressed HIV-associated neurocognitive disorder. AIDS 2016;30: 591-600 CrossRef Medline

17. Cysique LA, Maruff P, Darby D, et al. The assessment of cognitive function in advanced HIV-1 infection and AIDS dementia complex using a new computerised cognitive test battery. Arch Clin Neuropsychol 2006;21:185-94 CrossRef Medline

18. Lane T, Moore D, Batchelor J, et al. Facial emotional processing in HIV infection: relation to neurocognitive and neuropsychiatric status. Neurosychology 2012;26:713-22 CrossRef Medline

19. Goebel R, Esposito, F. Formisano E. Analysis of functional image analysis contest (FIAC) data with BrainVoyager QX: from singlesubject to cortically aligned group general linear model analysis and self-organizing group independent component analysis. Hum Brain Mapp 2006;27:392-401 CrossRef Medline

20. Goshtasby A. Piecewise linear mapping functions for image registration. Pattern Recog 1986;19:459-66 CrossRef

21. Weissenbacher A, Kasess C, Gerstl F, et al. Correlations and anticorrelations in resting-state functional connectivity MRI: a quantitative comparison of preprocessing strategies. Neuroimage 2009;47: 1408-16 CrossRef Medline

22. Forman SD, Cohen JD, Fitzgerald M, et al. Improved assessment of significant activation in functional magnetic resonance imaging (fMRI): use of a cluster-size threshold. Magn Reson Med 1995;33: 636-47 CrossRef Medline

23. Bressler SL, Menon V. Large-scale brain networks in cognition: emerging methods and principles. Trends Cogn Sci 2010;14:277-90 CrossRef Medline

24. Kallianpur KJ, Kirk GR, Sailasuta N, et al. Regional cortical thinning associated with detectable levels of HIV DNA. Cereb Cortex 2012;22: 2065-75 CrossRef Medline

25. Gongvatana A, Harezlak J, Buchthal S, et al; HIV Neuroimaging Consortium. Progressive cerebral injury in the setting of chronic HIV infection and antiretroviral therapy. J Neurovirol 2013;19:209-18 CrossRef Medline

26. Amboni M, Tessitore A, Esposito F, et al. Resting-state functional connectivity associated with mild cognitive impairment in Parkinson's disease. J Neurol 2015;262:425-34 CrossRef Medline

27. Dennis EL, Thompson MP. Functional brain connectivity using fMRI in aging and Alzheimer's disease. Neuropsychol Rev 2014;24: 49-62 CrossRef Medline

28. Liu C, $\mathrm{Li} \mathrm{C}$, Yin X, et al. Abnormal intrinsic brain activity patterns in patients with subcortical ischemic vascular dementia. PLoS One 2014;9:e87880 CrossRef Medline

29. Castellazzi, G, Palesi, Casiraghi L, et al. Patterns of altered functional connectivity in Alzheimer disease and vascular dementia. In: Proceedings of the Annual Meeting and Exhibition of the International Society for Magnetic Resonance in Medicine, Toronto, Ontario. Canada. May 30-June 5, 2015

30. Brier $\mathrm{MR}, \mathrm{Wu} \mathrm{Q}$, Tanenbaum $\mathrm{AB}$, et al. Effect of HAART on brain organization and function in HIV-negative subjects. J Neuroimmune Pharmacol 2015;10:517-21 CrossRef Medline

31. Marra CM, Zhao Y, Clifford DB, et al; Clinical Trials Group 736 Study Team. Impact of combination antiretroviral therapy on cerebrospinal fluid HIV RNA and neurocognitive performance. AIDS 2009;23: 1359-66 CrossRef Medline

32. Bogoch II, Davis BT, Venna N. Reversible dementia in a patient with central nervous system escape of human immunodeficiency virus. J Infect 2011;63:236-39 CrossRef Medline 